\title{
On the dielectric approximation in the additional light waves theory: an historical rehabilitation (recovery)
}

\author{
V.N. Piskovoi, E.F. Venger \\ V. Lashkaryov Institute of Semiconductor Physics, NAS of Ukraine, \\ 41, prospect Nauky, 03028 Kyiv, Ukraine
}

\begin{abstract}
After the basic Pekar papers on crystal optics with spatial dispersion (CSD) among a lot of others there was the "dielectric approximation" (DA) method. But soon it has been fully rejected because, as it was recognized by the main group of authors on the theme, it came in conflict with the law of conservation of energy flux through the ideal vacuum-crystal interface. The objective of this paper is to advance a way for rehabilitation of DA method by using indispensable broadening and essential generalization of the expression just for the energy flux density vector (EFDV) at the vacuum-medium interface (the so-called Poynting-Pekar vector in the additional light waves (ALW) theory).
\end{abstract}

Keywords: additional light waves theory, dielectric approximation.

Manuscript received 02.02.15; revised version received 22.05.15; accepted for publication 03.09.15; published online 30.09.15.

For the sake of simplicity and clearness, we shall restrict ourselves to a simple model of polarization oscillators in a medium as well as the simplest geometrical configuration of the light-medium system. Also, we shall stand here in the framework of the exciton effective mass (EEM) approximation (the exciton effective mass $M$ is taken positive for the sake of definiteness).

Let us consider a case that a monochromatic plane wave (with the frequency $\omega$ and wave vector $k_{0}=\omega / c$, where $c$ is the speed of light in vacuum) is incident from vacuum along the normal to the surface of a semiinfinite optically uniaxial crystal (occupying the halfspace $x \geq 0$ ). We assume that this wave is in resonance with some nondegenerate dipole-allowed excitonic state to which a polarization vector $\mathbf{P}(0,0, P(x))$ corresponds.

The $\mathbf{P}$ vector, being directed along the crystal axis $C(Z)$, is parallel to that of the wave electric field $\mathbf{E}(0,0, E(x))$ and determines the partial contribution from the state studied into the total crystal polarization.
The ideal Maxwell equation in a medium has the standard form [1]

$\frac{\partial^{2} E}{\partial x^{2}}+k_{0}^{2}\left(\varepsilon_{0} E+4 \pi P\right)=0$,

where $\varepsilon_{0}$ is the background permittivity.

As it was shown in our papers [2], the material equations appropriate for the situation considered may be written in one of the following forms:

1. In the pioneer Pekar form [3] that arises from supplemented material Maxwell equation in the $(0, \infty)$ half-space to the $(-\infty, \infty)$ space with further reducing to zero the remainder part $(-\infty, 0)$ in $x \geq 0$ space (in a more generalized case, it corresponds to $U_{p}=-1$ or $P\left(0^{+}\right)=0$ in the point 4 , see bellow).

2. In Tolpygo-Mashkevich form that is based on the simultaneous use of the material and ideal equations in the integral form [4]. We have checked, of course, identicity of this approach to 
the problem with that of the points 3,4 , and came to conclusion that there is no reasons to base the paper on the different formulations of the ideal Maxwell equations.

3. One more historical approach is based on the fundamental Hopfield-Thomas paper [5]. In this approach, the Maxwell material equations for the above presented configuration is determined by the following material equation:

$$
\frac{\hbar}{2 M} \frac{\partial^{2} P}{\partial x^{2}}+\left(\omega-\omega_{e x}+i \gamma\right) P=-\frac{\varepsilon_{0} \Delta}{4 \pi} E .
$$

Here, $\omega_{e x}$ and $\gamma$ are the resonance exciton frequency and damping constant, respectively; the parameter $\Delta$ is equal to the constant of the socalled "longitudinal - transverse" splitting of exciton line, which, in its turn, may be written in several possible equivalent forms (see Eq. (9) in mob4 of 2004).

According to the studies of such class of equations in mathematical physics, the linear in $P$ boundary condition for Eq. (2) is of the following form

$$
P\left(0^{+}\right)+\alpha \frac{\partial P}{\partial x}\left(0^{+}\right)=0,
$$

where $\alpha$, in general, is some rather arbitrary parameter that will be partly determined in the point 4.

4. In the simplest integral form with some free enough parameter $U(|U| \leq 1)$ that determine the exciton interaction with crystal surface

$$
P(x)=\int_{0}^{\infty}\left[\chi\left(x-x^{\prime}, \omega\right)+U \chi\left(x+x^{\prime}, \omega\right)\right] E\left(x^{\prime}\right) d x^{\prime},
$$

where $\chi\left(x-x^{\prime}, \omega\right)$ is the polarization operator for an infinite medium:

$$
\begin{aligned}
& \chi\left(x-x^{\prime}, \omega\right)=-i \frac{\varepsilon_{0} \Delta M r}{4 \pi \hbar} \exp \left(\frac{i}{r}\left|x-x^{\prime}\right|\right), \\
& \text { with } r=\frac{2 M}{\hbar}\left(\omega-\omega_{e x}+i \gamma\right)^{-\frac{1}{2}} \text {. }
\end{aligned}
$$

It is obvious that all the above approaches to the problem must be equivalent, and their parameters should be closely connected to one another. Namely, parameter

$$
U_{p}=-1
$$

corresponds to $\alpha=0$ in (3), i.e., is the main and the most often used Pekar (or Dirichlet) condition with $P\left(0^{+}\right)=0$

$U_{g}=1$

that corresponds to the Ginzburg (or Neumann) case [6] with $\alpha^{-1}=0$ in Eq. (3), and $\frac{\partial P}{\partial x}\left(0^{+}\right)=0$
$U_{s}=-\left[1+2 i \frac{\delta d}{r(C+\delta)}\right]$, with $C=-\frac{\hbar^{2}}{2 M d^{2}}$,

where $d$ is the lattice constant in the $x$ direction and $\delta$ is the energy shift of a molecular level on the surface as compared to that of the crystal bulk. The latter corresponds to the Sugakov case [7] with $\alpha=\delta d /(C+\delta)$ in Eq. (3) - that was the first in ALW theory representation of expression for $P$ in the form of Eq. (4).

All the above relations can be easily checked by using Eq. (4) and its differentiation with respect to $x$, for arbitrarily $x$ as well as for $x$ approaching to $0^{+}$.

In full compliance with the abovementioned, the DA case was already considered in papers [8] with

$U_{d a}=0$.

In terminology of the point 3 , it determines $\alpha$ in Eq. (3) uniquely:

$\alpha_{d a}=-i r$.

At the same time, if the exciton effective mass approximation is applied consistently, then the expression for the energy flux density is also well defined, and the possible (virtual) jump of time-averaged normal components of the energy flux density ${ }_{\Delta} S_{0}$ at the vacuum-medium interface is given by the expression known in crystal optics with spatial dispersion:

$$
{ }_{\Delta} S_{0}=i \frac{\hbar \omega_{e x}}{2 M \Delta \varepsilon_{0}}\left(P \frac{\partial P^{*}}{\partial x}-P^{*} \frac{\partial P}{\partial x}\right)_{0^{+}} .
$$

For the most cases of Eqs. (6) to (8) type, the necessary turning ${ }_{\Delta} S$ to zero was easily checked, so from the very beginning this fact does not call any doubt as to its general applicability ad hoc and, naturally, has been omitted at the calculation of reflection and passing of EM waves for all above situations, that is for all possible meanings of parameter $U$, including also the case $U_{d a}=0$.

But soon a big enough group of authors (see, e.g., $[3,6,9,10])$ who used these to simple calculations for $U=0$ has noted, that in this case the law of energy conservation violates and ${ }_{\Delta} S_{0}$ has a discontinuity at $0^{+}$. Namely, this fact results in total ignoring the DA method in ALW theory as inevitable cost of scientific production.

Now our aim is to reanimate it. To this end, we shall take the generalized expression for ${ }_{\Delta} S$ (see the papers [2]) including the undetermined for a moment expression for the density of the surface polarization current $\mathbf{j}\left(0,0, j_{0}\right)$. In this case, ${ }_{\Delta} S$ takes the form:

${ }_{\Delta} S=-\frac{1}{4 \pi}\left[E_{0} j_{0}^{*}+c . c\right]+{ }_{\Delta} S_{0}$ 
with $j_{0}=\sigma(\omega) E_{0}$, and $\sigma$ as the one more free but not completely independent parameter, because it may be related with the parameter $U$ or $\alpha$ by the relation:

$\left({ }_{\Delta} S\right)_{0^{+}}=0$.

As it has been argued in [2], one can choose $\sigma$ in such a way that $\operatorname{Im}(\sigma)=0$ in the absence of real surface conductivity.

So, the boundary conditions for electric field and magnetic induction $B\left(-\frac{1}{C} \frac{\partial \mathbf{B}}{\partial t}=\operatorname{rot} \mathbf{E}\right)$, in the frame of the taken above space configuration and the time dependence of the fields, take the following form:

$E\left(0^{-}\right)=E\left(0^{+}\right)$,

$\frac{\partial E}{\partial x}\left(0^{-}\right)=\frac{\partial E}{\partial x}\left(0^{+}\right)+4 \pi \frac{i k_{0}}{c} j_{0}$.

Here, $0^{\mp}$ designates approaching the crystal boundary on the left and right to the origin of coordinates, respectively.

So, step by step we come to the complete system of consistent equations necessary for solving the assigned problem. As usually, we seek its solution in the framework of the plane waves, namely $P(x), E(x) \rightarrow\left(E_{j}, P_{j}\right) \exp \left(i k_{j} x\right) \quad$ with $\quad k_{j}=k_{0} n_{j}$ for each possible value of the wave-vector. We obtain two different roots for refraction, $n_{1}$ and $n_{2}$ in accordance with the second order in $x$ of two joint homogeneous Eqs. (1) and (2) and with the requirement for absence of any electromagnetic field sources at $(+\infty)$ - for halfspace configuration.

These two roots for refraction are

$n_{1,2}^{2} \equiv n_{+,-}^{2}=\frac{1}{2}\left(\mu+\varepsilon_{0}\right) \pm \sqrt{\frac{1}{4}\left(\mu-\varepsilon_{0}\right)^{2}+b}$

where $\mu \equiv \frac{2 M}{\hbar k_{0}{ }^{2}}\left(\omega-\omega_{e x}+i \gamma\right), b=\frac{2 M \Delta \varepsilon_{0}}{\hbar k_{0}^{2}}$. And, accordingly, the relations

$P_{j}=\frac{1}{4 \pi}\left(n_{j}^{2}-\varepsilon_{0}\right) E_{j}$,

$\frac{\partial P_{j}}{\partial x}\left(0^{+}\right)=i k_{0} n_{j} P_{j}\left(0^{+}\right)$.

As a result, we have the complete system of equations for the problem under consideration. Being written in terminology of $E_{i}, E_{R}, E_{1}, E_{2}$ for amplitudes of incident, reflected and two penetrating waves, it has the following form

$$
\left\{\begin{array}{l}
E_{i}+E_{R}=E_{1}+E_{2} \equiv E_{0} \\
E_{i}-E_{R}=n_{1} E_{1}+n_{2} E_{2}+\frac{4 \pi}{c} \sigma E_{0} \\
\sum_{j=1,2}\left(n_{j}^{2}-\varepsilon_{0}\right)\left(1+i \alpha k_{0} n_{j}\right) E_{j}=0
\end{array}\right.
$$

where $\quad \sigma=\frac{c}{4 \pi} k_{0} b \operatorname{Im}(\alpha)\left|n_{1}+n_{2}+i \alpha k_{0} f\right|^{-2} \quad$ (with $\left.f=n_{1}^{2}+n_{2}^{2}+n_{1} n_{2}-\varepsilon_{0}\right)$. It follows from Eq. (13) by a transformation from $P_{j}$ and $\frac{\partial P_{j}}{\partial x}$ to $E_{1,2}$ (see equations (17), (18)) and then via $E_{0}$ from the first and third equations of the system (19).

This fact makes it possible to express all fields arising in crystal and free space via the field $E_{0}$ and to construct the reflection amplitude coefficient $(R)$ for the situation considered:

$R \equiv \frac{E_{R}}{E_{i}}=\frac{1-n_{\text {eff }}}{1+n_{\text {eff }}}$,

where $n_{\text {eff }}$ is the effective refractive index:

$n_{\text {eff }}=\frac{n_{1} n_{2}+\varepsilon_{0}+i \alpha k_{0} n_{1} n_{2}\left(n_{1}+n_{2}\right)}{n_{1}+n_{2}+i \alpha k_{0} f}+\frac{4 \pi}{c} \sigma$

It is evident that different parts of the expression for $n_{\text {eff }}$ may be combined in many ways, but here we take those of them that can be easily compared with the simplest and well known ones, namely:

the Pekar [3] result $\left(\alpha=0, \sigma=0, P_{e x}\left(0^{+}\right)=0\right)$ with

$n_{\text {eff }}=\frac{n_{1} n_{2}+\varepsilon_{0}}{n_{1}+n_{2}}$

and the Ginzburg [6] result $\left(\alpha^{-1} \rightarrow 0, \sigma=0, \frac{\partial P_{e x}}{\partial x}\left(0^{+}\right)=0\right)$ with

$n_{\text {eff }}=n_{1} n_{2}\left(n_{1}+n_{2}\right) f^{-1}$.

So, we fulfilled the main aim of the paper. First, we have given the way for rehabilitation of DA and a group of other similar cases in the ALW theory, in principle. Second, we have given the correct expressions for the coefficients of reflection and refraction of polariton waves in DA for a specific "light - crystal" orientation (see Eqs. (20) and (21) with $i \alpha=r$, and $\sigma \neq 0$, in principle).

Some additional remarks.

After the first Pekar paper on the ALW problem, a lot of publications on the subject appeared. Many of them deal with different types of generalization (as to the variety of exciton-like states, so for their degeneracy or multiplicity, for oblique incidence with different $\mathrm{ABC}$ for different polarization vector projections, etc.) and can be found in the rather complete review [11], in the cited above papers [2] and elsewhere. But here we cited the really pioneer works only (see points $1-4$ above) which turned out enough for our aim.

The authors would like to thank the members of Pekar's seminar for helpful discussions on the theme and personally Dr. V. Korotyeyev for helpful discussions on the theme. 
References

1. L.D. Landau and E.M. Lifshits, Electrodynamics of Continuous Media, 2nd Ed. Pergamon Press, Oxford, 1984.

2. V.N. Piskovoi, Ya.M. Strelniker // Semiconductor Physics, Quantum Electronics and Optoelectronics, 1, p. 18 (1998); V.N. Piskovoi, E.F. Venger, Ya.M. Strelniker // Phys. Rev. B66, 115402 (2002); E.F. Venger, V.N. Piskovoi // Phys. Rev. B70, p. 1 (2004).

3. S.I. Pekar // Sov. Phys. JETP, 6, p. 785 (1958); S.I. Pekar, Crystal Optics and Additional Light Waves. The Benjamin/Cummings Publishing Company Menlo Park, California, 1983; Frontiers in Physics, 57, Addison-Wesley, Reading Mass.

4. V.S. Mashkevich // Zhurn. Eksp. Teor. Fiz. 38, p. 906 (1960); 40, p. 1803 (1961); 42, p. 133 (1962).

5. J.J. Hopfield and D.G. Thomas // Phys. Rev. 132, p. 563 (1963).
6. V.L. Ginzburg // Zhurn. Eksp. Teor. Fiz. 34, p. 1593 (1958); V.M. Agranovich and V.L. Ginzburg, Crystal Optics with Spatial Dispersion and Excitons. Springer, Berlin, Heidelberg, New York, 1984.

7. V.I. Sugakov // Fiz. Tverd. Tela 5, p. 2204 (1963); 5, p. 1959 (1964); 5, p. 2682 (1963), in Russian.

8. G.S. Agarwal, D.N. Pattanayak, E. Wolf // Phys. Rev. B10, p. 1447 (1974); B11, p. 1342 (1975); J.L. Birman, J.J. Sein // Phys. Rev. B6, p. 2482 (1972); A.A. Maradudin, D.L. Mills // Phys. Rev. B7, p. 278 (1973); J.T. Foley, A.J. Devaney // Phys. Rev. B12, p. 3104 (1975); P. Halevi, Spatial Dispersion in Solids and Plasmas. North-Holland, Amsterdam, 1992.

9. M.F. Bishop, A.A. Maradudin // Phys. Rev. B14, p. 3384 (1976).

10. A. Selkin // phys. status solidi, 83, p. 47 (1977).

11. A. Stahl, I. Balslev, Electrodynamics of the Semiconductor Band Edge // Springer Tracts in Modern Physics, 110. 